\title{
QTLs for stomatal and photosynthetic traits related to salinity tolerance in barley
}

\author{
Xiaohui Liu ${ }^{1,2+}$, Yun Fan ${ }^{3 \dagger}$, Michelle Mak', Mohammad Babla', Paul Holford', Feifei Wang ${ }^{1,3}$, Guang Chen ${ }^{4}$, \\ Grace Scott', Gang Wang ${ }^{2}$, Sergey Shabala ${ }^{3}$, Meixue Zhou ${ }^{3^{*}}$ and Zhong-Hua Chen ${ }^{1^{*}}$
}

\begin{abstract}
Background: Stomata regulate photosynthesis and transpiration, and these processes are critical for plant responses to abiotic stresses such as salinity. A barley double haploid population with 108 lines derived from a cross between CM72 (salt-tolerant) and Gairdner (salt-sensitive) was used to detect quantitative trait loci (QTLs) associated with stomatal and photosynthetic traits related to salinity tolerance.

Results: A total of 11 significant QTLs (LOD > 3.0) and 11 tentative QTLs $(2.5<\mathrm{LOD}<3.0)$ were identified. These QTLs are distributed on all the seven chromosomes, except $5 \mathrm{H}$ and explain 9.5-17.3\% of the phenotypic variation. QTLs for biomass, intercellular $\mathrm{CO}_{2}$ concentration, transpiration rate and stomatal conductance under control conditions co-localised together. A QTL for biomass also co-located with one for transpiration rate under salinity stress. A linkage was found between stomatal pore area and gas exchange. A QTL for salinity tolerance also co-localised with QTLs for grain yield and biomass on chromosome 3H. Based on the draft barley genome, the candidate genes for salinity tolerance at this locus are proposed.
\end{abstract}

Conclusions: The lack of major QTLs for gas exchange and stomatal traits under control and saline conditions indicates a complex relationship between salinity and leaf gas exchange due to the fact that these complex quantitative traits are under the control of multiple genes.

Keywords: Gas exchange, Guard cell, Hordeum vulgare L, QTL mapping, Stomatal regulation

\section{Background}

Soil salinity results from natural causes such as from soluble salts from rocks and oceanic salts carried in wind and rain as well as from increasing salinization of agricultural land due to irrigation and deforestation $[1,2]$. Salinity is causing major global food security issues due to the large arable area that is now saline and not suitable for cropping; therefore, breeding salt tolerant crops has become a top priority. Genetic modification to produce transgenic plants containing novel genes or different expression levels of existing genes can improve plant salt tolerance [3]. However, salinity tolerance is controlled by multi-gene traits, where genes are expressed

\footnotetext{
* Correspondence: meixue.zhou@utas.edu.au; Z.Chen@westernsydney.edu.au ${ }^{\dagger}$ Equal contributors

${ }^{3}$ School of Land and Food and Tasmanian Institute of Agriculture, University of Tasmania, Hobart, TAS 7249, Australia

'School of Science and Health, Hawkesbury Institute for the Environment, Western Sydney University, Penrith, NSW 2751, Australia

Full list of author information is available at the end of the article
}

at a number of plant developmental stages in a highly tissue-specific manner. Genetic engineering of single genes has proven problematic for improving salt tolerance in crops [4], and it is unlikely that salt tolerance could be improved by manipulating the expression of only one or few genes $[2,5]$. However, molecular breeding could be used to breed salt tolerant crops by exploiting existing genetic variation through direct selection or markerassisted selection in conjunction with the use of quantitative trait loci (QTLs) for gene pyramiding.

Stomata are formed by two highly specialised guard cells, and some are surrounded by subsidiary cells in certain plant species like barley [6]. Stomata control the exchange of water vapour and $\mathrm{CO}_{2}$ between the leaf interior and the atmosphere, and serve as major gateways for $\mathrm{CO}_{2}$ influx into plants as well as transpirational water loss from plants [7-9]. Transpirational water loss through stomatal pores accounts for $70 \%$ of total water loss indicating their significance in water use [10]. The stomatal aperture is influenced by the plant 
and its environment [11]. Under saline conditions, plant cells lose water and reduce cell elongation for short-term osmotic adjustment and later build up cellular $\mathrm{NaCl}$ over a longer period $[2,12]$. The accumulation of $\mathrm{NaCl}$ in plant cells, including stomatal guard cells, affects their function. Stomatal closure is one of the most immediate responses to salinity $[2,13,14]$, and this response is believed to be crucial for minimising plant water loss under hyperosmotic conditions in their rhizosphere [15-17]. Reducing stomatal density is another way of optimising the balance between leaf water loss and $\mathrm{CO}_{2}$ assimilation. Halophytes, naturally salt-tolerant species, are capable of reducing stomatal density when grown under hypersaline conditions $[18,19]$. The same effect has been observed in most tolerant barley varieties [20]. However, this strategy has a cost, as a reduction in stomata will reduce photosynthesis thereby reducing plant biomass and crop yield [21].

Stomatal and photosynthetic parameters, such as stomatal size and frequency, stomatal conductance, carbon assimilation, transpiration rate and water use efficiency, affect the grain yields of crops under stressed and non-stressed conditions [22, 23]. Genotypic variation in stomatal traits has been reported, but little is known about the genetic mechanisms behind these traits. A negative association between water loss and stomatal size was found in durum wheat [24], and while Wang and Clarke's [25] growth-room experiment reported a positive correlation between stomatal density and the rate of water loss in excised wheat leaves. Stomatal paremeters (e.g., stomatal aperture, guard cell volume, aperture width and aperture width/length) were significantly different between salt-tolerant and salt-sensitive genotypes. Significant correlations have been found between stomatal traits, expression of slow anion channel genes and grain yield in salt-tolerant barley [26]. This suggests that stomatal traits may contribute to salinity tolerance in barley, but further study using genetically different populations is required.

Many studies on the salinity tolerance of plants focus on ionic relations, but there has been little research to determine the potential role of stomatal function in salinity tolerance. QTLs for gas exchange and stomatal parameters under greenhouse conditions or different stresses have been identified in Arabidopsis [27], rice [8, 28, 29], sunflower [30], and faba bean [31]. In barley, QTLs associated with net photosynthetic rate have been detected under drought stress [32] and without stress [33], and QTLs for stomatal conductance [33] and stomatal density [34] have been identified in barley grown without stress. However, to the best of our knowledge, QTLs for stomatal traits, especially stomatal aperture and guard cell and subsidiary cell geometry under salinity stress, have not been reported in plants.

In our recent work, we have explored stomatal and photosynthetic traits as potential selection criteria for plant salt tolerance [26]. Here, we measured stomatal and photosynthetic traits in a double haploid (DH) population of barley to identify significant QTLs. We hypothesised that stomatal traits are controlled by multiple genes and would result in multiple QTLs for salt tolerance in barley. Thus, the objectives of this study were to: (1) identify QTLs for stomatal and photosynthetic traits associated with salinity tolerance in barley, and (2) investigate the relationships between salinity tolerance and stomatal regulation through QTL mapping.

\section{Methods}

\section{Plant materials and growth conditions}

A barley DH population consisting of 108 lines from a cross between CM72 (salt-tolerant) and Gairdner (saltsensitive) were used. Seeds of these lines, the two parental cultivars and two reference cultivars (Yerong (salt-tolerant) and Franklin (salt-sensitive)) were conducted at the Hawkesbury Campus of Western Sydney University, Australia. Seeds ( 5 per pot) were germinated and grown in $4 \mathrm{~L}$ pots containing potting mix augmented with $5 \mathrm{~g}$ Osmocoat $^{\ominus}$ slow release fertiliser (Debco Pty Ltd, Victoria, Australia). Two parallel trials were conducted in two glasshouse rooms with grow lamps $(600 \mathrm{~W})$ at a temperature of $25 \pm 1{ }^{\circ} \mathrm{C}, 65 \%$ relative humidity $(\mathrm{RH})$ and a light/dark photoperiod of $12 / 12 \mathrm{~h}$. Prior to treatment with $\mathrm{NaCl}$, all plants were watered twice weekly and fertilised with Hoagland's solution. The plants were subjected to $\mathrm{NaCl}$ treatment beginning at Week 5 after sowing at a rate of $50 \mathrm{mM} \mathrm{NaCl}$ per day over four consecutive days delivering a stepped final concentration of $200 \mathrm{mM} \mathrm{NaCl}$ in an attempt to avoid osmotic shock. All leached salt was collected in a saucer under the pot and re-applied to ensure the stability of concentrations across all treatment pots. Control plants were watered every day. The soil electrical conductivity (EC) was measured regularly with a portable EC meter (HI 991301, HANNA Instruments, Italy). Four weeks after salt treatment, gas exchange and stomatal assays were conducted. Grain yield and biomass were determined at Week 20. In addition, three glasshouse trials evaluating the salinity tolerance of the CM72/Gairdner DH population were conducted at Launceston, Tasmania, Australia. Plant growth conditions and salt treatment were similar to those previously described [35].

\section{Gas exchange measurements}

Gas exchange measurements were made according to O'Carrigan et al. [36] to determine net photosynthetic rate, intercellular $\mathrm{CO}_{2}$ concentration, stomatal conductance, transpiration rate, leaf vapour pressure deficit and leaf temperature. Measurements were taken over six consecutive days using an infrared gas analyser (model LI-6400XT, Li-Cor Inc., Lincoln, NE, USA), using the third fullyexpanded leaves of seedlings four weeks after the salt treatment ended. The measuring chambers had an air flow rate 
of $500 \mathrm{~mol} \mathrm{~s}^{-1}$, saturating photosynthetically active radiation (PAR) of $1500 \mu \mathrm{mol} \mathrm{m}{ }^{-2} \mathrm{~s}^{-1}$, a $\mathrm{CO}_{2}$ concentration of $400 \mu \mathrm{mol} \mathrm{mol}{ }^{-1}$ and relative humidity of $65 \%$. Gas exchange measurements were taken at the same time (approximately 10 a.m. to 4 p.m.) as those for stomatal assays.

\section{Measurement of stomatal parameters}

Twelve stomatal traits were analysed as described by Liu et al. [26], Mak et al. [37] and O'Carrigan et al. [36]. The parameters were aperture length (AL), aperture width (AW), aperture width/length (AWL), stomatal pore area (SA), guard cell length $(\mathrm{GCL})$, guard cell width $(\mathrm{GCW})$, guard cell volume (GCV), subsidiary cell length (SCL), subsidiary cell width (SCW), subsidiary cell volume (SCV), stomatal density (SD) and stomatal index (SI). For these measurements, the third fully expanded leaves were collected from the glasshouse and placed on tissue paper soaked in a stabilising solution $(50 \mathrm{mM} \mathrm{KCl}$, $5 \mathrm{mM} \mathrm{Na}^{+}$-MES, pH 6.1) in Petri dishes. Abaxial epidermal strips were then peeled and mounted on slides using a measuring solution $\left(10 \mathrm{mM} \mathrm{KCl}, 5 \mathrm{mM} \mathrm{Ca}^{2+}\right.$-MES, $\mathrm{pH}$ 6.1). Quick peeling and mounting was important to ensure stomatal images were true representations of the stomata found naturally on the whole plant in the glasshouse. Stomatal imaging was conducted using a CCD camera (NIS-F1 Nikon, Tokyo, Japan) attached to a microscope (Leica Microsystems AG, Solms, Germany). All images were analysed using Nikon NIS Element imaging software (Nikon, Tokyo, Japan) and measured with Image J software (NIH, USA).

\section{Salinity tolerance score}

Salt tolerance score was assessed at the seedling stage by combining scores for leaf chlorosis and plant survival $(0=$ no damage and $10=$ all dead $)$ when the most susceptible lines showed severe symptoms [38]. In this study, salt tolerance evaluation was based on the average value from results obtained in 2010, 2014 and 2015 in Launceston, Australia.

\section{QTLs and statistical analysis}

The data regarding photosynthetic and stomatal traits, biomass and grain yield, measured under control and saline conditions, were used for QTL analysis. The ratios of these traits in saline to control conditions were also tested for QTL identification. A genetic linkage map for this population was constructed using 886 markers including 868 Diversity Array Technology (DArT) and 18 Simple Sequence Repeat (SSR) markers. The software package, MapQTL 6.0 [39], was used to detect QTLs. QTLs were first analysed by interval mapping (IM). Following this, the closest marker at each putative QTL identified with interval mapping was selected as a cofactor, and the selected markers were used as genetic background controls in the approximate multiple QTL model (MQM). Logarithm of the odds (LOD) threshold values, applied to declare the presence of a QTL, were estimated by performing genome-wide permutation tests implemented in MapQTL version 6.0 using 1000 permutations of the original data set for each trait, resulting in a 95\% LOD threshold of around 3.0. To determine the effects of other traits on the QTLs for salinity tolerance, the QTLs for salinity tolerance were re-analysed using other traits as covariates. Two LOD support intervals around each QTLs were established, by taking the two positions, left and right of the peak, that had LOD values of two less than the maximum [39], after performing restricted MQM mapping which does not use markers close to the QTL. The percentage of variance explained by each QTL $\left(R^{2}\right)$ was obtained using restricted MQM mapping implemented with MapQTL 6.0. Graphical representation of linkage groups and QTLs was carried out using MapChart 2.2 [40]. Frequency distribution analysis was performed using SigmaPlot 12 (Systat Software Inc., San Jose, CA, USA). Skewness analysis was conducted. If skewness is less than -1 or greater than +1 , the distribution is highly skewed; if skewness is between -1 and $-1 / 2$ or between $+1 / 2$ and +1 , the distribution is moderately skewed, and if skewness is between $-1 / 2$ and $+1 / 2$, the distribution is approximately symmetric [41].

\section{Genomic analysis of potential genes for salinity tolerance} The sequence marker, Bmac0209, associated with the QTL for salinity tolerance score on $3 \mathrm{H}$ was used to identify candidate genes for salinity tolerance. The genome sequence of this region was retrieved by following a BLAST search (http://webblast.ipk-gatersleben.de/barley/). A morex_contig, 84335, was found to be homologous with Bmac0209. The physical map position of this contig was located at $51.77 \mathrm{cM}$ on $3 \mathrm{H}$. Barley genomic data and gene annotations were downloaded from ftp://ftpmips.helmholtz-muench en.de/plants/barley/public_data/ [42] and ftp://ftpmips. helmholtz-muenchen.de/plants/barley/public_data/popseq_ IPK/ [43]. Annotated genes between 46.74 and $56.72 \mathrm{cM}$ were deemed to be potential genes for salinity tolerance (Additional file 1: Table S1).

\section{Results}

The DH and parental lines show a large diversity in salinity tolerance

Significant differences in stomatal and photosynthetic traits between parental line CM72 and Gairdner were described in Liu et al. [26]. The $108 \mathrm{DH}$ lines showed significant differences in salinity tolerance, with CM72 being scored as 1 and Gairdner at 5 (Fig. 1a). Grain yield, biomass, leaf temperature, transpiration rate, stomatal area 

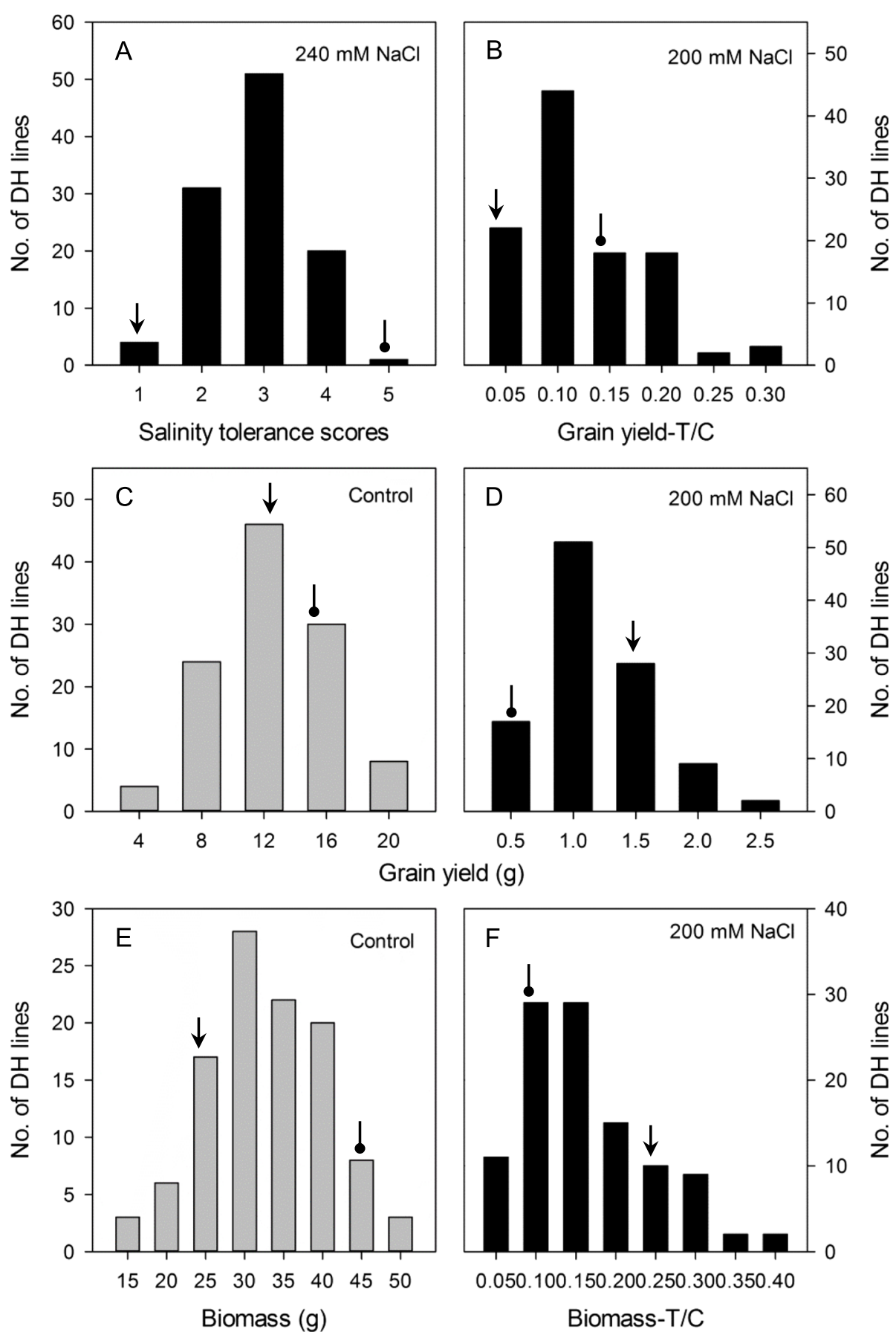

Fig. 1 Frequency distribution for salinity tolerance score $(\mathbf{a})$, relative grain yield-T/C (b), grain yield $(\mathbf{c}$, $\mathbf{d})$, biomass $(\mathbf{e})$ and relative biomass-T/C (f) of DH lines derived from the cross of CM72 and Gairdner under control and salt treatment. T/C: the ratio of traits under salt treatment (T) and control (C). Arrow represents CM72 and oval arrow represents Gairdner. Data are averages of four replicates. Salinity tolerance score are averaged over three years with three replicates each year

and other parameters of the $\mathrm{DH}$ lines under control or saline conditions displayed continuous frequency distributions (Figs. 1, 2 and 3). Of all 26 traits listed in Figs. 1, 2 and 3, 17 showed approximately symmetric distribution, six moderately skewed and three highly skewed
(Additional file 2: Table S2). Salinity stress caused a significant shift in the distribution of photosynthetic and stomatal traits and in grain yields (Figs. 1, 2 and 3). Stomatal conductance, transpiration rate and intracellular $\mathrm{CO}_{2}$ concentration showed a distribution skewed to 

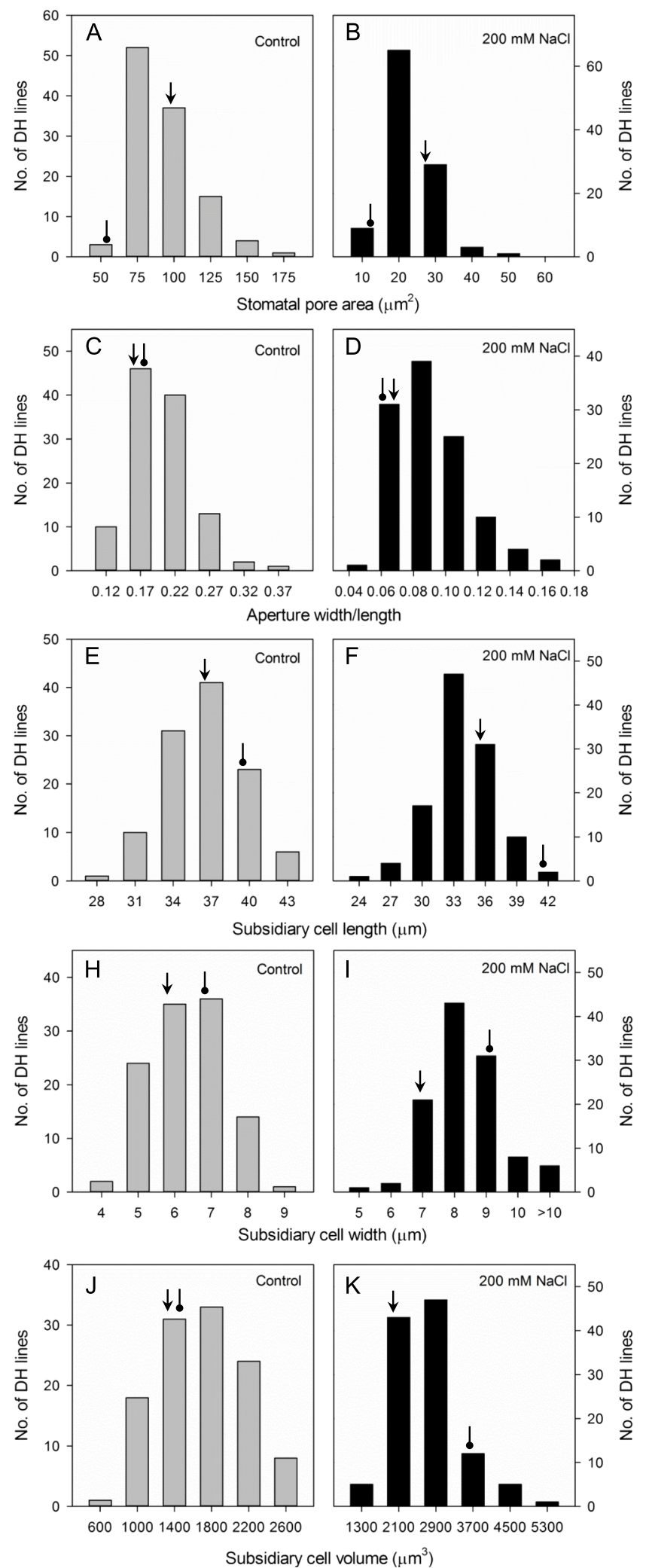

Fig. 2 (See legend on next page.) 
(See figure on previous page.)

Fig. 2 Frequency distribution of stomatal traits in control and salt treatment. Shown are stomatal pore area (a, b), aperture width/length (c, d), subsidiary cell length $(\mathbf{e}, \mathbf{f})$, subsidiary cell width $(\mathbf{h}, \mathbf{i})$ and subsidiary cell volume $\mathbf{j}, \mathbf{k})$ of DH lines derived from the cross of CM72 and Gairdner, under control and salinity treatment conditions. Arrow represents CM72 and oval arrow represents Gairdner. Data are averages of 16-73 cells from 4 replicates

lower values under salt stress, whereas leaf vapour pressure deficit and leaf temperature displayed a distribution skewed to higher ranges (Fig. 3). For stomatal traits, stomatal pore area, aperture width/length and subsidiary cell length had distributions skewed to lower values under salt stress; in contrast, subsidiary cell width and subsidiary cell volumes showed a distribution skewed to higher ranges (Fig. 2). Grain yields were skewed to lower values under salinity treatment. Of the two parental lines, CM72 showed better performance than Gairdner for all traits under saline stress (Figs. 1, 2 and 3). This enabled the QTL mapping to identify a total of 11 QTLs (Fig. 4, Table 1 and Additional file 3: Figure S1 with LOD values of $>3.0$ Additional file 4: Figure S2).

\section{Significant QTLs for gas exchange and stomatal traits under control and saline conditions}

One QTL for transpiration rate under control conditions (QTR-C.CmGa.1H) was detected on chromosome $1 \mathrm{H}$ close to bpb-1381 and explained 14\% of the phenotypic variation. Two QTLs associated with leaf temperature were identified at the same position on chromosome $2 \mathrm{H}$, QLT-C.CmGa.2H under control conditions and QLT-T.CmGa.2H in the salinity treatment, respectively explaining 16.5 and $11.2 \%$ of the phenotypic variation (Fig. 4, Table 1 and Additional file 4: Figure S2). Only one significant QTL, QSA-T.CmGa.1H associated with stomatal pore area under salinity treatment, was found on the long arm of chromosome $1 \mathrm{H}$; this had a LOD value of 3.12. This QTL, with the closest marker being bPb-9081, accounted for $12.5 \%$ of the phenotypic variation (Fig. 4, Table 1 and Additional file 4: Figure S2).

\section{Significant QTLs for grain yield, biomass and salinity tolerance under control and saline conditions}

Four QTLs for grain yield were found on chromosomes $1 \mathrm{H}, 2 \mathrm{H}$ and 3H: QGY-T.CmGa.1H and QGY-T.CmGa.3H under salinity treatment; QGY-C.CmGa.2H under control conditions; and QGY-T/C.CmGa.3H based on the ratio of salinity stress relative to control. QGY-C.CmGa.2H (with Ebmatc0039 as its nearest marker) explained $15.0 \%$ of the phenotypic variation and QGY-T.CmGa.1H (with bPb7043 as its closest marker) accounted for $13.9 \%$ of the phenotypic variation (Fig. 4, and Table 1). In addition, two QTLs for biomass were identified on chromosomes $1 \mathrm{H}$ and $2 \mathrm{H}$. The QTL, QBM-C.CmGa.1H, found under control conditions and flanked by bPb-1381, explained $15 \%$ of the phenotypic variation. In contrast, QBM-T.CmGa.2H, found under salinity treatment and with the nearest marker being bPb-7803, is located near the telomere of the short arm of chromosome $2 \mathrm{H}$ and explained $14 \%$ of the phenotypic variation (Fig. 4 and Table 1). Furthermore, QST.CmGa.3H (60.1 cM), which is associated with salinity tolerance, was identified on chromosome $3 \mathrm{H}$ with Bmac209 being the nearest marker. This QTL explained $16.8 \%$ of phenotypic variation and had a LOD value of 4.29 (Fig. 4 and Table 1).

\section{Tentative QTL for gas exchange traits under control and saline conditions}

Apart from the significant QTLs, 11 tentative QTLs $(2.5<\mathrm{LOD}<3.0)$ were also identified in this study (Additional file 3: Figure S1 and Table 1). A QTL (GS-C) for stomatal conductance and one for intercellular $\mathrm{CO}_{2}$ concentration (CI-C) under control conditions were located on chromosome $1 \mathrm{H}$ near marker bPb-1381 (Additional file 3: Figure $\mathrm{S} 1$ and Table 1). There are three tentative QTLs on chromosome $2 \mathrm{H}$ that are associated with stomatal conductance under saline conditions (GS-T), transpiration rate (TR-T/C) and vapour pressure deficit (VPD-T/C) found under salinity stress relative to control (Additional file 3: Figure S1 and Table 1). Also, a QTL for leaf temperature under salt treatment (LT-T) and one (LT-C) under control conditions were located on chromosomes $5 \mathrm{H}$ and $7 \mathrm{H}$, respectively (Additional file 3: Figure S1 and Table 1). In addition, four tentative QTL were identified for stomatal traits. One QTL for aperture width/length under salt treatment (AWL-T) was found on chromosome $3 \mathrm{H}$ (Additional file 3: Figure S1 and Table 1). Another QTL for subsidiary cell length under salt treatment (SCL-T) was identified on chromosome $4 \mathrm{H}$, while QTLs for subsidiary cell width $(\mathrm{SCW}-\mathrm{C})$ and subsidiary cell volume $(\mathrm{SCV}-\mathrm{C})$ under control conditions were both found on chromosome $6 \mathrm{H}$ (Additional file 3: Figure $\mathrm{S} 1$ and Table 1).

\section{Co-localisation of phenotypic traits}

There are five clusters of QTLs for different traits (Fig 4 and Additional file 3: Figure S1). On chromosome 1H, QTLs for transpiration rate (TR-C), biomass (BM-C), stomatal conductance (GS-C) and intercellular $\mathrm{CO}_{2}$ concentration (CI-C) under control conditions were located at the same position $(137.6 \mathrm{cM})$ and shared a common nearest marker, bpb-1381 (Table 1). On chromosome 

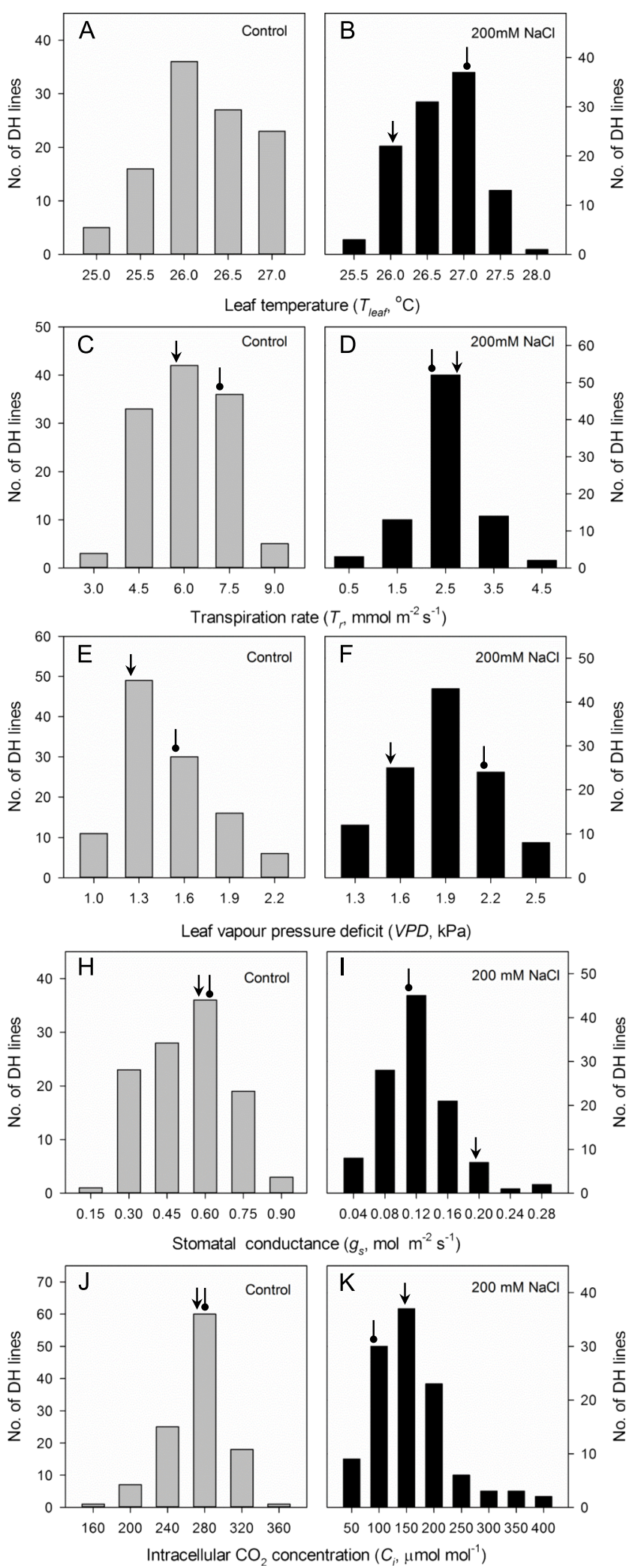

Fig. 3 Frequency distribution of gas exchange traits in control and salt treatment. Shown are leaf temperature $(\mathbf{a}, \mathbf{b})$, transpiration rate $(\mathbf{c}$, d), leaf vapour pressure deficit $(\mathbf{e}, \mathbf{f})$, stomatal conductance $(\mathbf{h}, \mathbf{i})$ and intracellular CO2 concentration $(\mathbf{j}, \mathbf{k})$ of DH lines derived from the cross of CM72 and Gairdner. Arrow represents CM72 and oval arrow are Gairdner. Data are averages of four replicates 
$1 \mathrm{H}$

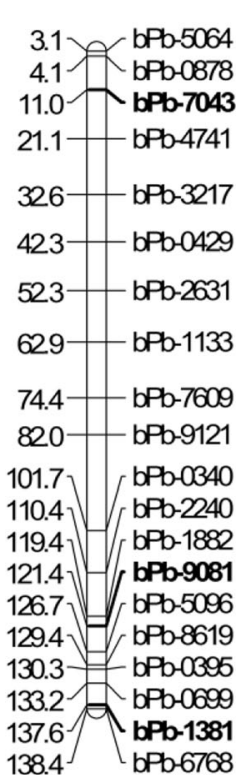

2H

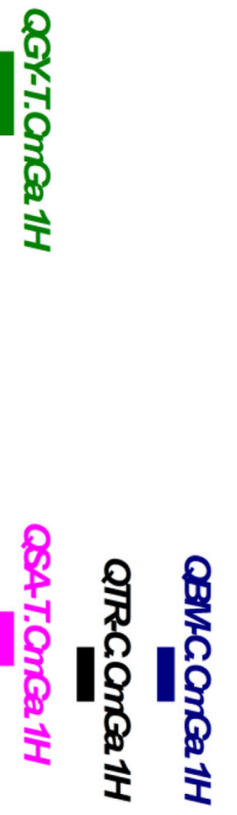

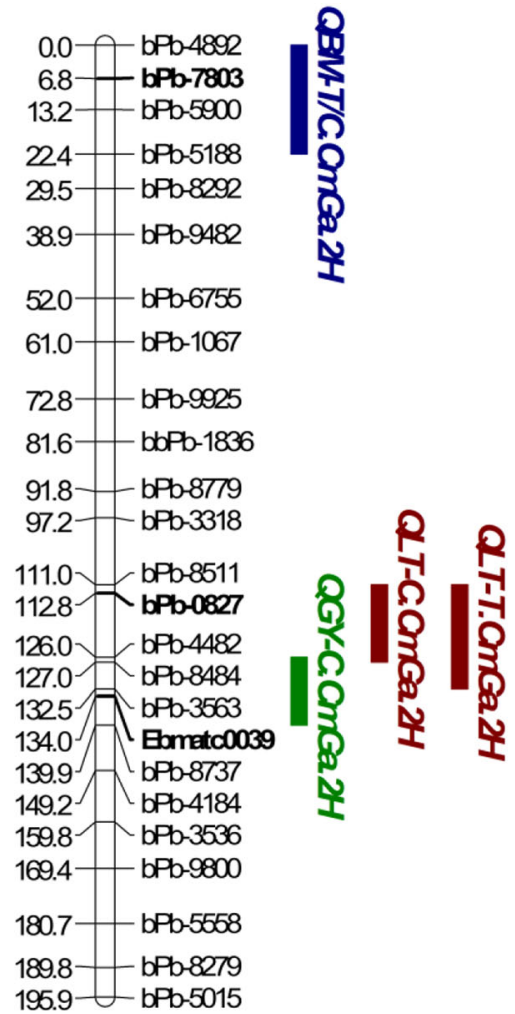

3H

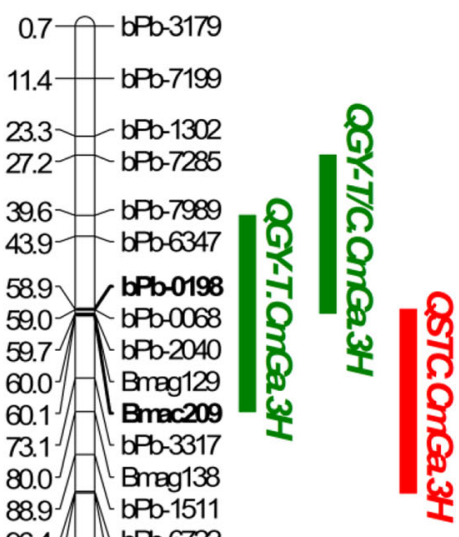

Fig. 4 QTLs associated with salinity tolerance (in red), grain yield (in green), biomass (in blue), stomatal area (in pink), transpiration rate (in black) and temperature of leaves (in brown). For clarity, only part of the chromosome regions which cover 2-LOD interval of all the QTLs are shown. C: Control; T: salt treatment

$2 \mathrm{H}$, two QTLs for biomass and transpiration rate relative to control $(\mathrm{Bm}-\mathrm{T} / \mathrm{C}, \mathrm{TR}-\mathrm{T} / \mathrm{C})$ were both at $6.8 \mathrm{cM}$ with bpb-7803 being the closest marker, while another QTL for stomatal conductance under salt treatment (GS-T) was located at $10.3 \mathrm{cM}$ close to QBM-T/C.CmGa.2H and QTR-T/C.CmGa.2H (Table 1). In addition, QTLs for leaf temperature under control (LT-C) and saline conditions (LT-T) were at same position $(112.8 \mathrm{cM})$ on chromosome $2 \mathrm{H}$ with bpb-0827 as the closest marker (Table 1). Furthermore, QTLs for grain yield (GY-T, GY-T/C) on chromosome $3 \mathrm{H}(58.9 \mathrm{cM})$ were located close to a QTL for salinity tolerance score $(60.1 \mathrm{cM})$ (Fig. 5 and Table 1). Also, QTLs for subsidiary cell width (SCW-C) and subsidiary cell volume $(\mathrm{SCV}-\mathrm{C})$ under control conditions were both at $93.5 \mathrm{cM}$ on chromosome $6 \mathrm{H}$ with EBmac602 as the nearest marker (Table 1).

\section{Identification of candidate genes for salinity tolerance on} $3 \mathrm{H}$

QTLs for grain yield relative to the control were located close to the QTL for salinity tolerance score on chromosome $3 \mathrm{H}$ (Figs. 4 and 5; Table 1). This led to further investigation of the possible genes controlling salinity tolerance in barley. Annotated genes close to SSR marker, Bmac0209, were examined from the published sequence of the barley genome. There is a number of potential salt tolerance-related genes within this region of interest (Additional file 1: Table S1). These genes related to: reactive oxygen species (ROS) detoxification, including a peroxidase (AK249079.1 at $51.63 \mathrm{cM}$ ) and a respiratory burst oxidase-like protein (MLOC_81745.1 at $50.67 \mathrm{cM}$ ); ion transport, including a potassium channel (MLOC_74879.2 at $51.27 \mathrm{cM})$, an outward rectifying potassium channel (MLOC_18521.1 at $55.1 \mathrm{cM}$ ), a vacuolar cation/proton exchanger (MLOC_13658.1 at $51.35 \mathrm{cM}$ ), a V-type proton ATPase (AK251977.1 at $51.63 \mathrm{cM}$ ), a voltage-gated chloride channel (MLOC_57123.4 at 56.44 cM); and some transcription factors possibly involved in guard cell signal transduction such as Myb domain protein MYB (MLOC_7981.1 at $51.63 \mathrm{cM}), \mathrm{WD}-40$ repeat protein (AK370701 at $51.63 \mathrm{cM})$, $\mathrm{Ca}^{2+}$ dependent protein kinase CDPK (MLOC_12765.1 at $47.04 \mathrm{cM}), \mathrm{Ca}^{2+}$ independent protein kinase CIPK (MLOC_9827.2 at $49.29 \mathrm{cM}$ ) and calcineurin-B like activator CBL (MLOC_60474.3 at $55.17 \mathrm{cM}$ ). 
Table 1 QTL and tentative QTL identified for different traits under saline and control conditions in a double haploid population derived from a cross between CM72 and Gairdner

\begin{tabular}{|c|c|c|c|c|c|c|}
\hline Trait & QTL & Linkage group & Position & LOD & $R^{2}(\%)$ & Nearest marker \\
\hline GY-T & QGY-T.CmGa.1H & $1 \mathrm{H}$ & 11 & 3.95 & 12.5 & bPb-7043 \\
\hline SA-T & QSA-T.CmGa.1H & $1 \mathrm{H}$ & 121.4 & 3.12 & 12.5 & bPb-9081 \\
\hline TR-C & QTR-C.CmGa.1H & $1 \mathrm{H}$ & 137.6 & 3.52 & 14 & bPb-1381 \\
\hline$B M-C$ & QBM-C.CmGa.1H & $1 \mathrm{H}$ & 137.6 & 3.79 & 15 & bPb-1381 \\
\hline GS-C & & $1 \mathrm{H}$ & 137.6 & 2.76 & 11.2 & bPb-1381 \\
\hline $\mathrm{Cl}-\mathrm{C}$ & & $1 \mathrm{H}$ & 137.6 & 2.75 & 11.2 & bPb-1381 \\
\hline BM-T/C & QBM-T.CmGa.2H & $2 \mathrm{H}$ & 6.8 & 3.49 & 14 & bPb-7803 \\
\hline TR-T/C & & $2 \mathrm{H}$ & 6.8 & 2.54 & 10.4 & bPb-7803 \\
\hline GS-T & & $2 \mathrm{H}$ & 10.3 & 2.54 & 10.3 & bPb-1949 \\
\hline LT-C & QLT-C.CmGa.2H & $2 \mathrm{H}$ & 112.8 & 4.19 & 16.5 & bPb-0827 \\
\hline LT-T & QLT-T.CmGa.2H & $2 \mathrm{H}$ & 112.8 & 3.09 & 11.2 & bPb-0827 \\
\hline GY-C & QGY-C.CmGa.2H & $2 \mathrm{H}$ & 134 & 3.77 & 15 & Ebmatc0039 \\
\hline VPD-T/C & & $2 \mathrm{H}$ & 153.6 & 2.63 & 10.7 & bPb-2701 \\
\hline GY-T & QGY-T.CmGa.3H & $3 \mathrm{H}$ & 58.9 & 4.35 & 13.9 & bPb-0198 \\
\hline GY-T/C & QGY-T/C.CmGa.3H & $3 \mathrm{H}$ & 58.9 & 4.42 & 17.3 & bPb-0198 \\
\hline STC & QSTC.CmGa.3H & $3 \mathrm{H}$ & 60.1 & 4.29 & 16.8 & Bmac209 \\
\hline AWL-T & & $3 \mathrm{H}$ & 136.9 & 2.78 & 11.3 & bPb-3634 \\
\hline SCL-T & & $4 \mathrm{H}$ & 122.5 & 2.98 & 12 & bPb-6153 \\
\hline LT-T & & $5 \mathrm{H}$ & 53.3 & 2.69 & 9.7 & bPb-2762 \\
\hline SCW-C & & $6 \mathrm{H}$ & 93.5 & 2.81 & 11.4 & EBmac602 \\
\hline SCV-C & & $6 \mathrm{H}$ & 93.5 & 2.58 & 10.5 & EBmac602 \\
\hline LT-C & & $7 \mathrm{H}$ & 63.9 & 2.81 & 9.5 & bPb-1209 \\
\hline
\end{tabular}

Tentative QTL: $2.5<$ LOD $<3.0$. QTL: LOD $>3.0$

Abbreviations: $-C$ traits under control conditions, $-T$ traits under salt treatment, $-T / C$ the ratio of traits under salt treatment to traits under control conditions, $G Y$ grain yield, $B M$ biomass, STC salinity tolerance score, gas exchange traits, $T R$ transpiration rate, $L T$ leaf temperature, $G S$ stomatal conductance, $C l$ intercellular $C_{2}$ concentration, VPD leaf vapour pressure deficit, SA stomatal pore area, AWL aperture width/length, SCL subsidiary cell length, SCW subsidiary cell width, SCV subsidiary cell volume

\section{Discussion and conclusion}

Intercellular $\mathrm{CO}_{2}$ concentration, transpiration rate and stomatal conductance are genetically linked to biomass production in barley

Salinity tolerance in plants including barley is inherently complex, controlled by polygenic traits and is affected by various mechanisms influencing photosynthesis [44-46]. Under control conditions, QTLs for intercellular $\mathrm{CO}_{2}$ concentration $(\mathrm{CI}-\mathrm{C})$, transpiration rate $(\mathrm{TR}-\mathrm{C})$ and stomatal conductance (GS-C) were closely located together with that for biomass (BM-C) on chromosome 1H (Additional file 3: Figure S1 and Table 1). QTLs associated with photosynthetic traits have rarely been reported in barley due to measurement procedures and the complicated, dynamic processes of these phenotypic traits. Liu et al. [33] identified several QTLs located on chromosomes $2 \mathrm{H}, 3 \mathrm{H}$ and $7 \mathrm{H}$ associated with intercellular $\mathrm{CO}_{2}$ concentration, transpiration rate and stomatal conductance from barley flag leaves on plants grown under normal conditions. QTLs for net photosynthetic rate, transpiration rate and stomatal conductance have also been found in rice [29]. Therefore, photosynthetic parameters have the potential to indicate salinity tolerance in grasses [47]. In our experiment, co-localisation of QTLs for gas exchange traits with those for biomass production demonstrated that intercellular $\mathrm{CO}_{2}$ concentration, transpiration rate and stomatal conductance are genetically linked to biomass production in barley. Moreover, QTLs for relative transpiration rate (TR-T/C) and relative biomass $(\mathrm{BM}-\mathrm{T} / \mathrm{C})$ contributing to growth and yield under salt stress were identified at the same position on chromosome $2 \mathrm{H}$ (Additional file 3: Figure S1 and Table 1).

$\mathrm{NaCl}$-induced accumulation of ABA in leaves leads to stomatal closure and reduced transpiration rate, thereby contributing to increased water use efficiency in plants [8]. Initial stomatal closure can serve as a rapid and effective response to salinity; however, long-term stomatal closure will limit $\mathrm{CO}_{2}$ uptake, photosynthesis and plant growth [27]. $\mathrm{CO}_{2}$ and water availability strongly influence 


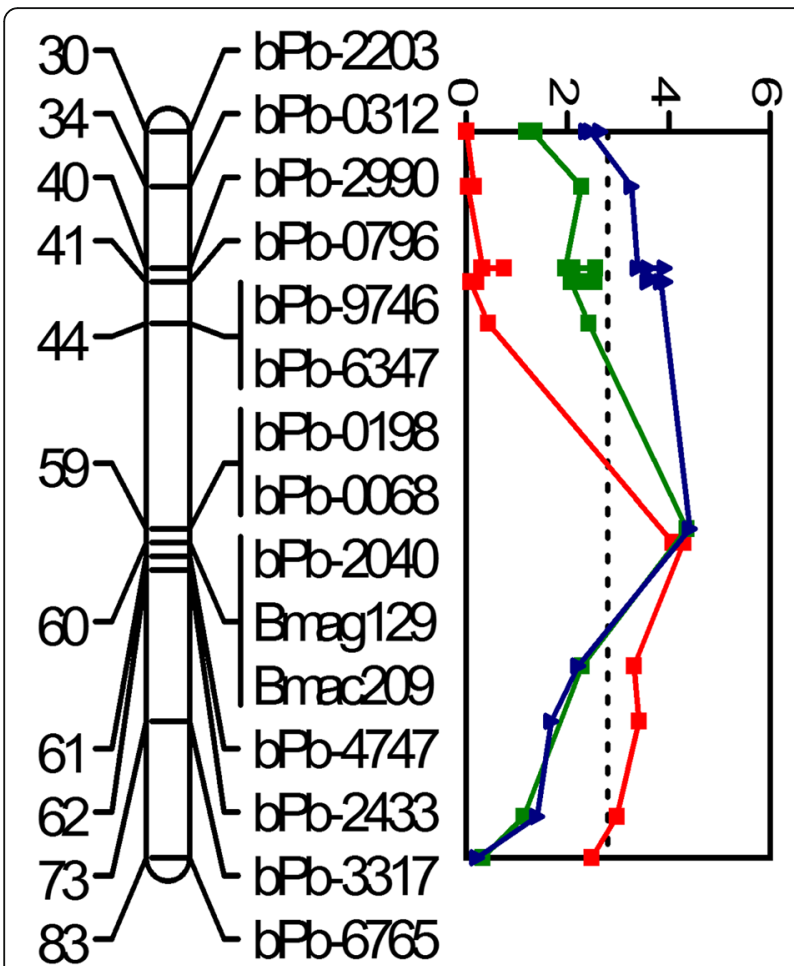

Fig. 5 QTL associated with salinity tolerance (red solid line), grain yield-T (green solid line) and grain yield-T/C (blue solid line) located at similar position on $3 \mathrm{H}$. The dotted line around LOD 3.0 is a line of significance. T: salt treatment; $T / C$ : the ratio of traits under salt treatment (T) and control (C)

stomatal opening and closure. Stomatal opening or closure directly affect stomatal conductance which further influences $\mathrm{CO}_{2}$ intake and transpirational water loss [7]. Short-term, elevated $\mathrm{CO}_{2}$ concentrations provoke stomatal closure, whereas long-term, elevated $\mathrm{CO}_{2}$ concentrations decrease stomatal density leading to reductions in transpiration [7, 48]. Stomatal conductance significantly influences net photosynthetic rate and is one of the key parameters limiting photosynthesis in barley [49]. In our study, we found genetic evidence for the importance of photosynthetic traits for barley production under salinity stress. Therefore, promoting leaf photosynthetic capacity and genetic modification of traits on which photosynthesis relies, are important approaches to enhancing crop biomass [50].

\section{Linkage between gas exchange and stomatal traits under salinity stress}

Gas exchange characteristics are influenced by stomatal structure, aperture and density [10]. Salt tolerance was associated with lower stomatal density and decreased stomatal area in Chenopodium quinoa [18], and a positive correlation between stomatal frequency and transpiration rate was reported in barley [51]. In rice, it was reported that high stomatal density was associated with high photosynthetic rate in Indica cultivars, while Japonica cultivars had higher transpiration efficiency [52]. In addition, it has been suggested that salinity may have a relatively direct impact on the photosynthetic apparatus independent of that on stomata [53]. Moreover, a remarkably negative correlation between stomatal density and size was found in lowland rice but no common QTL was found for these traits [8]. One of the aims of this study was to find potential QTLs connecting stomatal and photosynthetic traits for salinity tolerance in barley. In this study, a stomatal trait QTL for stomatal pore area (SA) was associated with the gas exchange traits of, transpiration rate (TR), stomatal conductance (GS) and intercellular $\mathrm{CO}_{2}$ concentration (CI) (Additional file 3: Figure S1). Conversely, the remaining stomatal trait QTLs for aperture width/length (AWL) and subsidiary cell length (SCL) did not show links with the gas exchange traits QTLs for leaf temperature (LT) and vapour pressure deficit (VPD). Therefore, our findings suggest that stomatal traits and gas exchange traits are not genetically well-linked. One potential reason could be that gas exchange measurements determine leaf $\mathrm{CO}_{2}$ assimilation and $\mathrm{H}_{2} \mathrm{O}$ transpiration, which are governed by stomatal and non-stomatal factors. However, the stomatal traits measured in this study may have little influence over the non-stomatal factors such as the genetic control of photosynthetic machinery $[9,10,48]$.

QTLs and candidate genes for salinity tolerance score and grain yield

Salinity tolerance score, assessed through the combination of plant survival and leaf wilting, has been used for evaluating barley salt tolerance in our previous studies $[35,38,54]$. Crop yield under salinity stress is a result of balancing resource allocation between growth and defence against stress, since responding to stress is deleterious to growth and yield [55]; therefore, the ability to produce high grain yield in saline soils is the ultimate criterion of salinity tolerance. In this study, we identified one QTL controlling salt tolerance score (QST.CmGa.3H) using plant survival and leaf wilting as an evaluation index. This QTL, located at $60.1 \mathrm{cM}$ on chromosome $3 \mathrm{H}$ (with Bmac209 as the nearest marker) explained $16.8 \%$ phenotypic variation. Interestingly, the QTL for grain yield relative to the control (QGY-T/C.CmGa.3H) was only $1.2 \mathrm{cM}$ away from this QTL for salinity tolerance score (Fig. 1). Therefore, we attempted to identify candidate genes for salinity tolerance on chromosome $3 \mathrm{H}$ using the published sequence of the barley genome (Additional file 1: Table S1). These candidate genes included those involved in ROS detoxification, photosynthesis, ion transport and signal transduction. Plants can perceive stress through transmembrane osmo-receptors and transduce the perception of environmental stimuli via internal signalling pathways. 
Induced transcription factors (TFs) and post-translational regulation of TFs lead to the expression of functional, downstream response genes associated with ion channels, secondary metabolite biosynthesis, ROS detoxification, stomatal closure, growth regulation, cell death as well as those encoding Late Embryogenesis Abundant (LEA) proteins [55]. Although we cannot rule out other genes in this region, these are candidates for further finemapping and functional analysis to verify their roles in salt tolerance in barley. Near isogenic lines are being developed in our current research work for fine mapping of these candidate genes.

\section{Co-localisation of QTLs associated with salinity tolerance}

Using various genetic populations, some QTLs for salinity tolerance in barley have been found and are associated with chlorophyll content, chlorophyll fluorescence, proline content, water soluble carbohydrates, relative water content [56], ion content $[57,58]$, and salinity tolerance [35, 38, 54, 59]. Previous QTL studies of barley salinity tolerance have lacked information on the genetic mechanisms underlying stomatal traits and gas exchange parameters. Interestingly, many QTLs for stomatal traits and gas exchange parameters (Figs. 2 and 3; Additional file 2: Table S2) co-localised with previously identified QTLs for agronomic or physiological traits (Fig. 4, Table 1 and Additional file 4: Figure S2). The co-localization of QTLs for stomatal conductance, leaf vapour pressure deficit, leaf temperature, chlorophyll content, chlorophyll fluorescence, water soluble carbohydrate and relative water content with salinity tolerance is not unexpected, because these traits are associated with photosynthesis and transpiration in barley. The relationship among these traits indicates that these parameters may not be independent but interacting. They may be co-regulated for the protection of photosynthetic apparatus, an important factor in tolerance to salinity stress [2]. In addition, the co-localisation of QTLs for stomatal conductance and leaf temperature under control or saline conditions could be linked with genes whose proteins control $\mathrm{K}^{+}, \mathrm{Na}^{+}$and $\mathrm{Cl}^{-}$homeostasis. Stomatal opening or closure is controlled by guard cells and adjacent subsidiary cells, and the 'shuttling' of ions and solutes between the two cell types [60] using channels and transporters to maintain ionic homoeostasis in these cells. The shuttle transport of $\mathrm{K}^{+}$between subsidiary and guard cells, resulting in prompt stomatal opening and closure. $\mathrm{K}^{+}$accumulation was generally detected in subsidiary cells during stomatal closure $[60,61] . \mathrm{K}^{+}$inward and outward rectifying channels and slow anion channels in guard cell could be responsive to ion shuttle transport within the stomatal complex [61-64]. Interestingly, six genes from the published barley genome sequence related with the transport of $\mathrm{K}^{+}$and anions are located close to QTL, QGY-T/C.CmGa.3H and to the SSR marker, Bmac0209
(Additional file 1: Table S1). Therefore, the fine mapping of genes encoding $\mathrm{K}^{+}$and $\mathrm{Cl}^{-}$channels should be performed followed by their functional analysis to verify their roles in salinity tolerance in barley.

\section{Additional files}

Additional file 1: Table S1. List of candidate genes within $10 \mathrm{cM}$ around Bmac0209 on chromosome 3H of barley. (XLSX 37 kb)

Additional file 2: Table S2. Skewness analysis of average data of all phenotypic traits from the DH lines used for QTL mapping in this study. (XLSX $106 \mathrm{~kb}$ )

Additional file 3: Figure S1. QTLS associated with different traits of the $\mathrm{DH}$ lines derived from the cross of CM72 and Gairdner under control and salinity conditions. (PPTX $429 \mathrm{~kb}$ )

Additional file 4: Figure S2. Distribution of QTLs discovered in this study (LOD value > 3.0). (PPTX $124 \mathrm{~kb}$ )

\section{Abbreviations}

AL: Aperture length; AW: Aperture width; AWL: Aperture width/length; $\mathrm{Cl}$ : Intercellular $\mathrm{CO}_{2}$ concentration; DArT: Diversity Array Technology; DH: Double haploid; GCL: Guard cell length; GCV: Guard cell volume; GCW: Guard cell width; GS: Stomatal conductance; IM: Interval mapping; LEA: Late Embryogenesis MBRYOGENESIS Abundant; LOD: Logarithm of the odds; LT: Leaf temperature; MQM: Multiple QTL model; PAR: Photosynthetically active radiation; QTLs: Quantitative trait loci; ROS: Reactive oxygen species; SA: Stomatal pore area; SCL: Subsidiary cell length; SCV: Subsidiary cell volume; SCW: Subsidiary cell width; SD: Stomatal density; SI: Stomatal index; SSR: Simple sequence repeat; TFs: Transcription factors; TR: Transpiration rate; VPD: Vapour pressure deficit

\section{Acknowledgements}

We thank Jennie Nelson, Linda Westmoreland, Elizabeth Kabanoff, Sumedha Dharmaratne, Rosemary Freeman, Renee Smith and Honglang Duan for their technical assistance. This work was supported by the Australian Department of Agriculture and an Australian Research Council DECRA (DE140101143) to Zhong-Hua Chen. Meixue Zhou and Sergey Shabala were supported by the funding from the Grains Research and Development Corporation. Xiaohui Liu was a recipient of China Scholarship Council award.

\section{Availability of data and materials}

The datasets supporting the conclusions of this article are included within the article and its additional files. The seeds of genetic lines in this study are maintained Tasmanian Institute of Agriculture, University of Tasmania.

\section{Authors' contributions}

$\mathrm{ZHC}$ and $\mathrm{MZ}$ designed research; $\mathrm{XL}, \mathrm{YF}, \mathrm{MM}$ and $\mathrm{MB}$ conducted the bulk of experiments with assistance from ZHC, MZ and FW; ZHC, MZ, SS, PH and GW supervised the experiments; XL, YF, ZHC, MZ, MM, MB, GC and GS analysed data; $\mathrm{ZHC}, \mathrm{XL}, \mathrm{YF}, \mathrm{MZ}, \mathrm{PH}$ and SS wrote the article with contributions of all the authors. All authors read and approved the final manuscript.

\section{Competing interests}

The authors declare that they have no competing interests.

\section{Consent for publication}

This original manuscript and any substantial parts of it are not under consideration by any other journal. All authors have read and approved the final manuscript for submission to BMC Genomics.

\section{Ethics approval and consent to participate}

Not applicable.

\section{Data deposition}

The dataset supporting the conclusions of this article is available in Research Data Storage at Western Sydney University and University of Tasmania. The data will be shared from the corresponding authors on request. 


\section{Author details}

${ }^{1}$ School of Science and Health, Hawkesbury Institute for the Environment, Western Sydney University, Penrith, NSW 2751, Australia. ${ }^{2}$ School of Environmental Science and Engineering, Tianjin University, Tianjin 300072, China. ${ }^{3}$ School of Land and Food and Tasmanian Institute of Agriculture, University of Tasmania, Hobart, TAS 7249, Australia. ${ }^{4}$ College of Agriculture and Biotechnology, Zhejiang University, Hangzhou 310058, China.

Received: 9 May 2016 Accepted: 6 December 2016

Published online: 03 January 2017

\section{References}

1. Rengasamy P. Transient salinity and subsoil constraints to dryland farming in Australian sodic soils: an overview. Anim Prod Sci. 2002;42(3):351-61.

2. Munns R, Tester M. Mechanisms of salinity tolerance. Annu Rev Plant Biol. 2008; $59: 651-81$

3. Cuin TA, Shabala S. Compatible solutes reduce ROS-induced potassium efflux in Arabidopsis roots. Plant Cell Environ. 2007;30(7):875-85.

4. Schroeder Jl, Delhaize E, Frommer WB, Guerinot ML, Harrison MJ, HerreraEstrella L, Horie T, Kochian LV, Munns R, Nishizawa NK. Using membrane transporters to improve crops for sustainable food production. Nature. 2013; 497(7447):60-6.

5. Flowers TJ. Improving crop salt tolerance. J Exp Bot. 2004;55(396):307-19.

6. Bergmann DC, Sack FD. Stomatal development. Annu Rev Plant Biol. 2007; 58:163-81.

7. Kim T-H, Boehmer M, Hu H, Nishimura N, Schroeder Jl. Guard Cell Signal Transduction Network: Advances in Understanding Abscisic Acid, $\mathrm{CO}_{2}$, and $\mathrm{Ca}^{2+}$ Signaling. Annu Rev Plant Biol. 2010;61:561-91.

8. Laza MRC, Kondo M, Ideta O, Barlaan E, Imbe T. Quantitative trait loci for stomatal density and size in lowland rice. Euphytica. 2010;172(2):149-58.

9. Lawson T, Blatt MR. Stomatal size, speed, and responsiveness impact on photosynthesis and water use efficiency. Plant Physiol. 2014;164(4):1556-70.

10. Hetherington AM, Woodward FI. The role of stomata in sensing and driving environmental change. Nature. 2003;424(6951):901-8.

11. Ainsworth EA, Rogers A. The response of photosynthesis and stomatal conductance to rising $\left[\mathrm{CO}_{2}\right]$ : mechanisms and environmental interactions. Plant Cell Environ. 2007;30(3):258-70.

12. Zhu JK. Salt and drought stress signal transduction in plants. Annu Rev Plant Biol. 2002;53:247-73.

13. Fricke W, Akhiyarova G, Veselov D, Kudoyarova G. Rapid and tissue-specific changes in $A B A$ and in growth rate in response to salinity in barley leaves. J Exp Bot. 2004;55(399):1115-23.

14. Fricke W, Akhiyarova G, Wei WX, Alexandersson E, Miller A, Kjellbom PO, Richardson A, Wojciechowski T, Schreiber L, Veselov D, et al. The short-term growth response to salt of the developing barley leaf. J Exp Bot. 2006;57(5): 1079-95.

15. Reef $R$, Lovelock CE. Regulation of water balance in mangroves. Ann Bot. 2015;115(3):385-95.

16. Shabala S, Pottosin I. Regulation of potassium transport in plants under hostile conditions: implications for abiotic and biotic stress tolerance. Physiol Plant. 2014;151(3):257-79.

17. El-Wahab A, El-Helw H, Tolba H. Physiological studies on the effect of inoculation with arbuscular mycorrhizae (AM) fungi on superior grape rootings under salt stress conditions. Nat Sci. 2011;9(1):85-100.

18. Shabala S, Hariadi Y, Jacobsen S-E. Genotypic difference in salinity tolerance in quinoa is determined by differential control of xylem $\mathrm{Na}+$ loading and stomatal density. Plant Physiol. 2013;170(10):906-14.

19. Shabala L, Mackay A, Tian Y, Jacobsen SE, Zhou D, Shabala S. Oxidative stress protection and stomatal patterning as components of salinity tolerance mechanism in quinoa (Chenopodium quinoa). Physiol Plant. 2012; 146(1):26-38.

20. Zhu M, Zhou M, Shabala L, Shabala S. Linking osmotic adjustment and stomatal characteristics with salinity stress tolerance in contrasting barley accessions. Funct Plant Biol. 2015:42(3):252-63.

21. Centritto $M$, Loreto $F$, Chartzoulakis $K$. The use of low $\left[\mathrm{CO}_{2}\right]$ to estimate diffusional and non-diffusional limitations of photosynthetic capacity of salt-stressed olive saplings. Plant Cell Environ. 2003;26(4):585-94.

22. Aminian R, Mohammadi S, Hoshmand S, Khodombashi M. Chromosomal analysis of photosynthesis rate and stomatal conductance and their relationships with grain yield in wheat (Triticum aestivum L.) under waterstressed and well-watered conditions. Acta Physiol Plant. 2011;33(3):755-64.
23. Khazaei H, Monneveux P, Hongbo S, Mohammady S. Variation for stomatal characteristics and water use efficiency among diploid, tetraploid and hexaploid Iranian wheat landraces. Genet Resour Crop Evol. 2010;57(2):307-14.

24. Venora G, Calcagno F. Study of stomatal parameters for selection of drought resistant varieties in Triticum durum DESF. Euphytica. 1991;57(3):275-83.

25. Wang $\mathrm{H}$, Clarke J. Relationship of excised-leaf water loss and stomatal frequency in wheat. Can J Plant Sci. 1993;73(1):93-9.

26. Liu X, Mak M, Babla M, Wang F, Chen G, Veljanoski F, Wang G, Shabala S, Zhou M, Chen Z. Linking stomatal traits and slow anion channel genes to grain yield for salinity tolerance in barley. Front Plant Sci. 2014;5:634.

27. Juenger TE, McKay JK, Hausmann N, Keurentjes JJB, Sen S, Stowe KA, Dawson TE, Simms EL, Richards JH. Identification and characterization of QTL underlying whole-plant physiology in Arabidopsis thaliana: delta C-13, stomatal conductance and transpiration efficiency. Plant Cell Environ. 2005; 28(6):697-708.

28. Price $A H$, Young EM, Tomos AD. Quantitative trait loci associated with stomatal conductance, leaf rolling and heading date mapped in upland rice (Oryza sativa). New Phytol. 1997;137(1):83-91.

29. Teng S, Qian Q, Zeng DL, Kunihiro Y, Fujimoto K, Huang DN, Zhu LH. QTL analysis of leaf photosynthetic rate and related physiological traits in rice (Oryza sativa L.). Euphytica. 2004;135(1):1-7.

30. Herve D, Fabre F, Berrios EF, Leroux N, Al Chaarani G, Planchon C, Sarrafi A, Gentzbittel L. QTL analysis of photosynthesis and water status traits in sunflower (Helianthus annuus L.) under greenhouse conditions. J Exp Bot. 2001;52(362):1857-64.

31. Khazaei H, O'Sullivan DM, Sillanpaa MJ, Stoddard FL. Use of synteny to identify candidate genes underlying QTL controlling stomatal traits in faba bean (Vicia faba L.). Theor Appl Genet. 2014;127(11):2371-85.

32. Wojcik-Jagla M, Rapacz M, Tyrka M, Koscielniak J, Crissy K, Zmuda K. Comparative QTL analysis of early short-time drought tolerance in Polish fodder and malting spring barleys. Theor Appl Genet. 2013;126(12):3021-34.

33. Liu L, Sun G, Ren X, Li C, Sun D. Identification of QTL underlying physiological and morphological traits of flag leaf in barley. BMC Genet. 2015;16(1):1

34. Patto MV, Rubiales D, Martin A, Hernandez P, Lindhout P, Niks R, Stam P. QTL mapping provides evidence for lack of association of the avoidance of leaf rust in Hordeum chilense with stomata density. Theor Appl Genet. 2003;106(7):1283-92

35. Zhou G, Johnson P, Ryan PR, Delhaize E, Zhou M. Quantitative trait loci for salinity tolerance in barley (Hordeum vulgare L.). Mol Breed. 2012;29(2):427-36.

36. O'Carrigan A, Hinde E, Lu N, Xu X-Q, Duan H, Huang G, Mak M, Bellotti B, Chen Z-H. Effects of light irradiance on stomatal regulation and growth of tomato. Environ Exp Bot. 2014;98:65-73.

37. Mak M, Babla M, Xu S-C, O'Carrigan A, Liu X-H, Gong Y-M, Holford P, Chen $\mathrm{Z}-\mathrm{H}$. Leaf mesophyll $\mathrm{K}^{+}, \mathrm{H}^{+}$and $\mathrm{Ca}^{2+}$ fluxes are involved in drought-induced decrease in photosynthesis and stomatal closure in soybean. Environ Exp Bot. 2014:98:1-12

38. Xu R, Wang J, Li C, Johnson P, Lu C, Zhou M. A single locus is responsible for salinity tolerance in a Chinese landrace barley (Hordeum vulgare L.). PLoS One. 2012;7(8):e43079.

39. Van Ooijen JW. MapQTL 6.0, software for the mapping of quantitative trait loci in experimental populations of dihaploid species. Wageningen: Kyazma BV; 2009.

40. Voorrips RE. MapChart: Software for the graphical presentation of linkage maps and QTLs. J Hered. 2002;93(1):77-8.

41. Bulmer MG. Principles of Statistics. New York: Dover Publications; 1979

42. Consortium IBGS. A physical, genetic and functional sequence assembly of the barley genome. Nature. 2012;491(7426):711-6.

43. Mascher M, Muehlbauer GJ, Rokhsar DS, Chapman J, Schmutz J, Barry K, Munoz-Amatriain M, Close TJ, Wise RP, Schulman AH, et al. Anchoring and ordering NGS contig assemblies by population sequencing (POPSEQ). Plant J. 2013:76(4):718-27.

44. Chen Z, Cuin TA, Zhou M, Twomey A, Naidu BP, Shabala S. Compatible solute accumulation and stress-mitigating effects in barley genotypes contrasting in their salt tolerance. J Exp Bot. 2007;58(15-16):4245-55.

45. Chen Z, Newman I, Zhou M, Mendham N, Zhang G, Shabala S. Screening plants for salt tolerance by measuring $\mathrm{K}^{+}$flux: a case study for barley. Plant Cell Environ. 2005;28(10):1230-46.

46. Chen Z, Zhou M, Newman IA, Mendham NJ, Zhang G, Shabala S. Potassium and sodium relations in salinised barley tissues as a basis of differential salt tolerance. Funct Plant Biol. 2007;34(2):150-62. 
47. Lee G, Carrow RN, Duncan RR. Photosynthetic responses to salinity stress of halophytic seashore paspalum ecotypes. Plant Sci. 2004;166(6):1417-25.

48. Lake JA, Woodward FI, Quick WP. Long-distance $\mathrm{CO}_{2}$ signalling in plants. J Exp Bot. 2002;53(367):183-93.

49. Jiang Q, Roche D, Monaco TA, Hole D. Stomatal conductance is a key parameter to assess limitations to photosynthesis and growth potential in barley genotypes. Plant Physiol. 2006;8(4):515-21.

50. Horton P. Prospects for crop improvement through the genetic manipulation of photosynthesis: morphological and biochemical aspects of light capture. J Exp Bot. 2000;51:475-85.

51. Miskin KE, Rasmusson DC, Moss DN. Inheritance and physiological effects of stomatal frequency in barley. Crop Sci. 1972;12(6):780-3.

52. Peng S, Laza RC, Khush GS, Sanico AL, Visperas RM, Garcia FV. Transpiration efficiencies of Indica and improved tropical Japonica rice grown under irrigated conditions. Euphytica. 1998;103(1):103-8.

53. Brugnoli E, Bjorkman O. Growth of cotton under continuous salinity stress: influence on allocation pattern, stomatal and non-stomatal components of photosynthesis and dissipation of excess light energy. Planta. 1992;187(3):335-47.

54. Fan Y, Shabala S, Ma Y, Xu R, Zhou M. Using QTL mapping to investigate the relationships between abiotic stress tolerance (drought and salinity) and agronomic and physiological traits. BMC Genomics. 2015;16(1):43.

55. Atkinson NJ, Urwin PE. The interaction of plant biotic and abiotic stresses: from genes to the field. J Exp Bot. 2012;63(10):3523-43.

56. Siahsar B, Narouei M. Mapping QTLs of physiological traits associated with salt tolerance in 'Steptoe' $\times$ 'Morex' doubled haploid lines of barley at seedling stage. J Food, Agric Environ. 2010;8(2):751-9.

57. Nguyen VL, Ribot SA, Dolstra O, Niks RE, Visser RG, van der Linden CG. Identification of quantitative trait loci for ion homeostasis and salt tolerance in barley (Hordeum vulgare L.). Mol Breed. 2013;31(1):137-52.

58. Xue D, Huang Y, Zhang X, Wei K, Westcott S, Li C, Chen M, Zhang G, Lance R. Identification of QTLS associated with salinity tolerance at late growth stage in barley. Euphytica. 2009;169(2):187-96.

59. Ma Y, Shabala S, Li C, Liu C, Zhang W, Zhou M. Quantitative trait loci for salinity tolerance identified under drained and waterlogged conditions and their association with flowering time in barley (Hordeum vulgare L). PLoS One. 2015;10(8):e0134822.

60. Mumm P, Wolf T, Fromm J, Roelfsema MRG, Marten I. Cell type-specific regulation of ion channels within the maize stomatal complex. Plant Cell Physiol. 2011;52(8):1365-75.

61. Raschke K, Fellows MP. Stomatal movement in Zea mays shuttle of potassium and chloride between guard cells and subsidiary cells. Planta. 1971;101:296-316

62. Chen ZH, Hills A, Lim CK, Blatt MR. Dynamic regulation of guard cell anion channels by cytosolic free $\mathrm{Ca}^{2+}$ concentration and protein phosphorylation. Plant J. 2010;61(5):816-25.

63. Chen Z-H, Hills A, Bätz U, Amtmann A, Lew VL, Blatt MR. Systems dynamic modeling of the stomatal guard cell predicts emergent behaviors in transport, signaling, and volume control. Plant Physiol. 2012;159(3):1235-51.

64. Wang Y, Papanatsiou M, Eisenach C, Karnik R, Williams M, Hills A, Lew VL, Blatt MR. Systems dynamic modeling of a guard cell $\mathrm{Cl}^{-}$channel mutant uncovers an emergent homeostatic network regulating stomatal transpiration. Plant Physiol. 2012;160(4):1956-67.

\section{Submit your next manuscript to BioMed Central and we will help you at every step:}

- We accept pre-submission inquiries

- Our selector tool helps you to find the most relevant journal

- We provide round the clock customer support

- Convenient online submission

- Thorough peer review

- Inclusion in PubMed and all major indexing services

- Maximum visibility for your research

Submit your manuscript at www.biomedcentral.com/submit

) Biomed Central 